\title{
Evidence-Based Oncology Practice: Competencies for Improved Patient Outcomes
}

\author{
KELLEY D. MAYDEN, MSN, FNP, AOCNP ${ }^{\circledR}$, IAC
}

From Legacy Wellmont Cancer Institute, Bristol, Virginia

Author's disclosures of conflicts of interest are found at the end of this article.

Correspondence to: Kelley D. Mayden, MSN, FNP, AOCNP, IAC, Legacy Wellmont Cancer Institute, Ballad Health, 349 Island Road, Bristol, VA 24201. E-mail: kdunrun@charter.net

https://doi.org/10.6004/jadpro.2019.10.1.7

(c) 2019 Harborside $^{\mathrm{TM}}$

\begin{abstract}
Oncology advanced practice providers (APP) play a critical role in providing cancer care to patients and families. Given the rate of scientific advancement, APPs are challenged to keep with the pace of science and acquire tools for successful practice. One such tool is evidencebased practice (EBP). Mastery of the competencies is obligatory and will assist APPs with the incorporation of EBP into clinical practice, and thus ensure the highest quality of patient care, forge the best patient outcomes, and reduce health-care expenditures. Advanced practice providers who achieve competency and proficiency have a professional responsibility to mentor others and assume leadership roles to help cement EBP as the standard of care.
\end{abstract}

oday, oncology advanced practice providers (APP) play a critical role in providing cancer care to millions of patients and families. This is a task most daunting given the volume of research dissemination, technological advancements, and the rising cost of cancer care, all in the age of precision medicine. In addition to patients and families, regulatory agencies and third-party payers demand error-free, high-quality care that translates into improved patient outcomes. One need only to examine the phenomenon of value-based contracting to understand these truths. With such rigorous demands from stakeholders, APPs must stock their practice arsenal with tools for successful practice.

\section{THE ROLE OF}

EVIDENCE-BASED PRACTICE One such tool is evidence-based practice (EBP). Evidence-based practice is a problem-solving approach to clinical decision-making that integrates the best research (external evidence), clinical expertise (internal evidence), and patient preferences and values (Melnyk, Fineout-Overholt, Gallagher-Ford, \& Kaplan, 2012). It is accepted both nationally and internationally as the current foundation for clinical 
practice. Research supports that the implementation of EBP ensures the highest quality of healthcare, improved patient outcomes, and decreased health-care cost (Melnyk, et al., 2012).

To understand its real importance, a statistic from a 2000 Institute of Medicine (IOM) report, To Err Is Human: Building a Safer Health System, stated that "at least 44,000 people, and perhaps as many as 98,000 people, die in hospitals each year as a result of medical errors that could have been prevented." In a follow-up report, Crossing the Quality Chasm: A New Health System For The $21^{\text {st }}$ Century, the IOM called for the reinvention of health-care systems to foster innovation and improve the delivery of care. One of the rules for design included decision-making that is evidencebased (IOM, 2001).

\section{FREQUENCY OF EVIDENCE-BASED PRACTICE IN THE US}

Although this may seem like rhetoric from an old sound bite, and despite its clear implications, EBP is not uniformly practiced in healthcare setting across the United States. In a crosssectional survey of 2,344 nurses in the United States from 19 hospitals or health-care systems, nurses reported they were not yet competent in meeting any of the 24 standard EBP competencies. Younger nurses and those with higher levels of education reported higher EBP competency (Melnyk et al., 2018). Although several factors may influence this lack of competency, mentorship-or the lack of it-may greatly influence EBP competency levels. In the same vein, ineffective leadership may impede the mastery of EBP competencies.

A descriptive survey conducted among 276 chief nurse executives (CNE) across the United States revealed that more than one third of hospitals are not meeting the National Database of Nursing Quality Indicators performance metrics, and almost one third of the hospitals are above national core measure benchmarks such as falls and pressure injuries (Melnyk et al., 2016). Although CNEs reported they believed in the value of EBP, their level of EBP implementation was low. They did not list EBP as a top priority, but they were concerned with patient safety and quality patient care. Little money was budgeted for implement- ing or sustaining EBP. It is interesting to note that the majority of hospitals maintained less than a $51 \%$ baccalaureate-prepared workforce, and only $18 \%$ of hospitals had Magnet status (Melnyk et al., 2016).

\section{Barriers to Evidence-Based Practice}

Although mandates for the use of EBP from assemblies such as the IOM, the U.S. Preventive Services Task Force, the Agency for Healthcare Research and Quality, the American Association of Colleges of Nursing, and the Institute for Healthcare Improvement should inspire the widespread adoption of EBP, a number of barriers preclude the universal adoption of EBP (see Table 1).

\section{CONCLUSION}

Advanced practice providers, with their role diversity and clinical expertise, are in a unique position to identify and eliminate barriers to EBP and help transform healthcare for the better. To achieve such a vital goal, APPs must commit to EBP, become familiar with the core competencies for EBP, and be willing to integrate them into practice. The truth is that many APPs are unfamiliar with the general competencies for EBP nursing as developed by the Quality and Safety Education for Nurses project. These competencies for APPs (see Table 2) build on and include the competencies for practicing registered nurses (see Table 3 ). Mastery of the competencies is obligatory and will assist APPs with the incorporation of EBP into clinical practice, and thus ensure the highest quality of patient care, forge the best patient outcomes, and reduce health-care expenditures. Advanced

\footnotetext{
Table 1. Barriers to the Implementation of Evidence-Based Practice

- Corporate barriers

- Financial constraints

- Lack of knowledge

- Lack of medical resources

- Lack of time

- Lack of training

- Negative attitudes about evidence-based practice
}

Note. Adapted from Sadeghi-Bazargani et al. (2014). 


\section{Table 2. Evidence-Based Practice Competencies} for Advanced Practice Nurses

- Systematically conducts an exhaustive search for external evidence (evidence generated from research) to answer clinical questions

- Critically appraises relevant preappraised evidence (e.g., clinical guidelines, summaries, synopses, syntheses of relevant external evidence) and primary studies, including evaluation and synthesis

- Integrates a body of external evidence from nursing and related fields with internal evidence (evidence generated internally within a clinical setting, such as patient assessment data, outcomes management, and quality improvement data) in making decisions about patient care

- Leads transdisciplinary teams in applying synthesized evidence to initiate clinical decisions and practice changes to improve the health of individuals, groups, and populations

- Generates internal evidence through outcomes management and EBP implementation projects for the purpose of integrating best practices

- Measures processes and outcomes of evidence-based clinical decisions

- Formulates evidence-based policies and procedures

- Participates in the generation of external evidence with other health-care professionals

- Mentors others in evidence-based decision-making and the EBP process

- Implements strategies to sustain an EBP culture

- Communicates best evidence to individuals, groups, colleagues, and policy makers

Note. EBP = evidence-based practice. Adapted from Melnyk et al. (2014)

practice providers who achieve competency and proficiency have a professional responsibility to mentor others and assume leadership roles to help cement EBP as the standard of care.

\section{Disclosure}

The author has no conflicts of interest to disclose.

\section{References}

Institute of Medicine. (2000). To err is human: Building a safer health system. Washington, DC: The National Academies Press. https://doi.org/10.17226/9728

Institute of Medicine. (2001). Crossing the quality chasm: $A$ new health system for the 21st century. Washington, DC: The National Academies Press. https://doi. org/10.17226/10027

Melnyk, B. M., Fineout-Overholt, E., Gallagher-Ford, L., \& Kaplan, L. (2012). The state of evidence-based practice in US nurses: Critical implications for nurse leaders and
Table 3. Evidence-Based Practice Competencies for Practicing Registered Nurses

- Questions clinical practices for the purpose of improving the quality of care

- Describes clinical problems using internal evidence (evidence generated internally within a clinical setting, such as patient assessment data, outcomes management, and quality improvement data)

- Participates in the formulation of clinical questions using the PICOT (patient population; intervention or area of interest; comparison intervention or group; outcome; time) format

- Searches for external evidence (evidence generated from research) to answer focused clinical questions

- Participates in the formulation of clinical questions using the PICOT format

- Searches for external evidence (evidence generated from research) to answer focused clinical questions

- Participates in critical appraisal of preappraised evidence (such as clinical practice guidelines, evidence-based policies and procedures, and evidence syntheses)

- Participates in the critical appraisal of published research studies to determine their strength and applicability to clinical practice

- Participates in the evaluation and synthesis of a body of evidence gathered to determine its strength and applicability to clinical practice

- Collects practice data (e.g., individual patient data, quality improvement data) systematically as internal evidence for clinical decision-making in the care of individuals, groups, and populations

- Integrates evidence gathered from external and internal sources in order to plan evidence-based practice changes

- Implements practice changes based on evidence and clinical expertise and patient preferences to improve care processes and patient outcomes

- Evaluates outcomes of evidence-based decisions and practice changes for individuals and groups

- Disseminates best practices supported by evidence to improve quality of care and patient outcomes and populations to determine best practices

- Participates in strategies to sustain an evidence-based practice culture

Note. Adapted from Melnyk et al. (2014)

educators. Journal of Nursing Administration, 42(9), 410417. https://doi.org/10.1097/NNA.0b013e3182664e0a

Melnyk, B. M., Gallagher-Ford, L., Long, L. E., \& FineoutOverholt, E. (2014). The establishment of evidencebased practice competencies for practicing registered nurses and advanced practice nurses in real-world clinical settings: Proficiencies to improve healthcare quality, reliability, patient outcomes, and cost. Worldviews on Ev- 
idence-Based Nursing, 11(1), 5-15. https://doi.org/10.1111/ wvn.12021

Melnyk, B. M., Gallagher-Ford, L., Thomas, B. K., Troseth, M., Wyngarden, K., \& Szalacha, L. (2016). A study of chief nurse executives indicates low prioritization of evidence-based practice and shortcomings in hospital performance metrics across the United States. Worldviews on Evidence-Based Nursing, 13(1), 6-14. https://doi. org/10.1111/wvn.12133

Melnyk, B. M., Gallagher-Ford, L., Zellefrow, C., Tucker, S.,
Thomas, B., \& Sinnott, L. T. (2018). The first U.S. study on nurses evidence-based practice competencies indicates major deficits that threaten healthcare quality, safety, and patient outcomes. Worldviews on EvidenceBased Nursing, 15(1), 16-25. https://doi.org/10.1111/ wvn.12269

Sadeghi-Bazargani, H., Tabrizi, J. S., \& Azami-Aghdash, S. (2014). Barriers to evidence-based medicine: A systematic review. Journal of Evaluation in Clinical Practice, 20(6), 793-802. https://doi.org/10.1111/jep.12222 